Pacific Journal of Mathematics

AN ANALOGUE OF PTOLEMY'S THEOREM AND ITS
CONVERSE IN HYPERBOLIC GEOMETRY 


\title{
AN ANALOGUE OF PTOLEMY'S THEOREM AND ITS CONVERSE IN HYPERBOLIC GEOMETRY
}

\author{
Joseph E. VALENTine
}

\begin{abstract}
The purpose of this paper is to give a complete answer to the question: what relations between the mutual distances of $n(n \geqq 3)$ points in the hyperbolic plane are necessary and sufficient to insure that those points lie on a line, circle, horocycle, or one branch of an equidistant curve, respectively?
\end{abstract}

In 1912 Kubota [6] proved an analogue of Ptolemy's Theorem in the hyperbolic plane and recently Kurnick and Volenic [7] obtained another analogue. In 1947 Haantjes [4] gave a proof of the hyperbolic analogue of the ptolemaic inequality, and in [5] he developed techniques which give a new proof of Ptolemy's Theorem and its converse in the euclidean plane. In the latter paper it is further stated that these techniques give proofs of an analogue of Ptolemy's Theorem and its converse in the hyperbolic plane. However, Haantjes' analogue of the converse of Ptolemy's Theorem is false, since Kubota [6] has shown that the determinant $\left|\sinh ^{2}\left(P_{i} P_{j} / 2\right)\right|$, where $i, j=1$, $2,3,4$, vanishes for four points $P_{1}, P_{2}, P_{3}, P_{4}$ on a horocycle in the hyperbolic plane. So far as the author knows, Haantjes is the only person who has mentioned an analogue of the converse of Ptolemy's Theorem in the hyperbolic plane.

Relations between the mutual distances of three points are obtained which are necessary and sufficient to insure that those points determine a line, circle, horocycle, or equidistant curve, respectively.

It will be recalled that Ptolemy's Theorem and its converse for the euclidean plane may be stated as follows.

Theorem (Ptolemy). Four points $P_{1}, P_{2}, P_{3}, P_{4}$ of the euclidean plane lie on a circle or line if and only if the determinant

$C\left(P_{1}, P_{2}, P_{3}, P_{4}\right)=\left|P_{i} P_{j}{ }^{2}\right|$ vanishes, where $P_{i} P_{j}=P_{j} P_{i}$ denotes the distance of the points $P_{i}, P_{j},(i, j=1,2,3,4)$.

The analogous theorem which will be proved in this paper is the following.

Theorem. Four points $P_{1}, P_{2}, P_{3}, P_{4}$ of the hyperbolic plane lie on a circle, line, horocycle, or one branch of an equidistant curve if and only if the determinant 
$\left|\sinh ^{2}\left(P_{i} P_{j} / 2\right)\right|$ vanishes $(i, j=1,2,3,4)$.

Moreover, the techniques used in this paper provide an easy extension of this analogue of Ptolemy's Theorem and its converse to give necessary and sufficient conditions for $n,(n \geqq 4)$, points to lie on a line, circle, horocycle, or equidistant curve.

2. Preliminary remarks. It is well known [1, pp. 273-274] that the determinants

$$
\Lambda_{4}\left(P_{1}, P_{2}, P_{3}, P_{4}\right)=\left|\cosh \left(P_{i} P_{j}\right)\right|,(i, j=1,2,3,4)
$$

and

$$
\Lambda_{5}\left(P_{1}, P_{2}, P_{3}, P_{4}, P_{5}\right)=\left|\cosh \left(P_{i} P_{j}\right)\right|,(i, j=1,2,3,4,5)
$$

vanish for each quadruple and each quintuple of points in the hyperbolic plane. Moreover, $\Lambda_{3}\left(P_{1}, P_{2}, P_{3}\right)=\left|\cosh \left(P_{i} P_{j}\right)\right| \geqq 0,(i, j=1,2,3)$ for each triple of points and vanishes if and only if the triple is collinear. These determinants play an important part in this paper.

Poincaré's circular model of the hyperbolic plane will be used.

3. Analogue of Ptolemy's Theorem and its converse. As was indicated in the $\S 2$, we will use Poincare's model of the hyperbolic plane. In order to prove the analogue of Ptolemy's Theorem and its converse, the "cross ratio" of four points is defined and shown to be an inversive invariant. Since lines, circles, horocycles, and equidistant curves are all equivalent under the group generated by hyperbolic inversions, this reduces Ptolemy's Theorem and its converse to that of considering four points on a line.

Definition 3.1. If $A, B, C, D$ are four distinct points, then the cross ratio $\{A B, C D\}$ is defined by :

$\{A B, C D\}=[\sinh A C / 2 \sinh B D / 2] /[\sinh A D / 2 \sinh B C / 2]$.

THEOREM 3.1. The cross ratio $\{A B, C D\}$ is an inversive invariant.

Proof. From the hyperbolic formula for inversion [8, p. 242], points $X, X^{\prime}$ are inverse points with respect to a circle with center $O$ and radius $2 \tanh ^{-1} \sqrt{k}$ if and only if $\tanh O X / 2 \tanh O X^{\prime} / 2=k$.

If $X^{\prime}, Y^{\prime}$ are the inverse points of $X, Y$, respectively, then the two triangles $O X Y$ and $O X^{\prime} Y^{\prime}$ have the same angle at $O$. It follows from the hyperbolic law of cosines that

$[\cosh O X \cosh O Y-\cosh X Y] /[\sinh O X \sinh O Y]=$ $\left[\cosh O X^{\prime} \cosh O Y^{\prime}-\cosh X^{\prime} Y^{\prime}\right] /\left[\sinh O X^{\prime} \sinh O Y^{\prime}\right]$. 
Let $x=\tanh O X / 2$ and $y=\tanh O Y / 2$. Then $\tanh O X^{\prime} / 2=k / x$, and consequently,

$$
\begin{gathered}
\cosh O X=\left(1+x^{2}\right) /\left(1-x^{2}\right), \sinh O X=2 x /\left(1-x^{2}\right), \\
\left.\cosh O X^{\prime}=\left(x^{2}+k^{2}\right) /\left(x^{2}-k^{2}\right), \sinh O X^{\prime}=2 x k /\left(x^{2}-k^{2}\right) \quad{ }^{*},{ }^{*}\right) \\
1+2 \sinh ^{2} X Y / 2=\cosh X Y .
\end{gathered}
$$

Substituting the values of $(*, *)$, together with the same identities when $x, X$ are replaced by $y, Y$, respectively, in $\left(^{*}\right)$ and solving the new equation for sinh $X^{\prime} Y^{\prime} / 2$, we obtain:

$$
\sinh X^{\prime} Y^{\prime} / 2=k\left[\left(1-x^{2}\right) /\left(x^{2}-k^{2}\right)\right]^{1 / 2}\left[\left(1-y^{2}\right) /\left(y^{2}-k^{2}\right)\right]^{1 / 2} \sinh X Y / 2 .
$$

Application of $(\tau)$ to all pairs of any four points $A, B, C, D$ yields,

$$
\left\{A^{\prime} B^{\prime}, C^{\prime} D^{\prime}\right\}=\{A B, C D\},
$$

which completes the proof of the theorem.

TheOREm 3.2. If $P_{1}, P_{2}, P_{3}, P_{4}$ are four points then the determinant $K\left(P_{1}, P_{2}, P_{3}, P_{4}\right)=\left|\sinh ^{2}\left(P_{i} P_{j} / 2\right)\right|$ is less than or equal to 0 , and $K\left(P_{1}, P_{2}, P_{3}, P_{4}\right)$ vanishes if $P_{1}, P_{2}, P_{3}, P_{4}$ lie on a line.

Proof. Since $\Lambda_{4}\left(P_{1}, P_{2}, P_{3}, P_{4}\right)=0$, it follows that the determinant $H$ obtained from $\Lambda_{4}\left(P_{1}, P_{2}, P_{3}, P_{4}\right)$, by bordering it with a first row and a first column with a common element -1 , and the rest of the elements in the first column all ones and the rest of the elements in the first row all zeroes, also vanishes.

The result of subtracting the first column of this bordered determinant from the second, third, fourth, and fifth columns, respectively, and making use of the fact that $\cosh (x)-1=2 \sinh ^{2}(x / 2)$ is

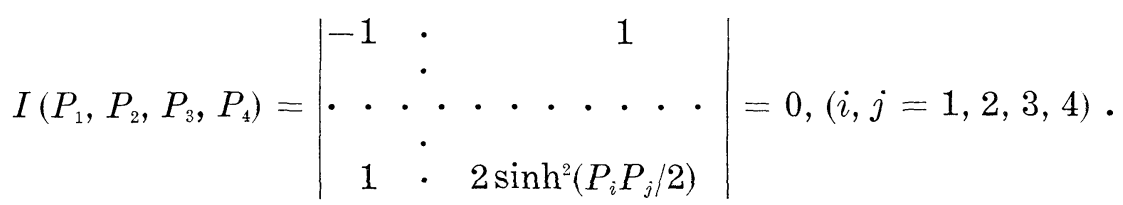

If the four points contain a nonlinear triple, assume the labeling so that $\Lambda_{3}\left(P_{2}, P_{3}, P_{4}\right) \neq 0$. Letting $[1,2]$ denote the cofactor of the element in the first row and second column, an expansion theorem from determinants yields

$$
-2^{4} K\left(P_{1}, P_{2}, P_{3}, P_{4}\right) \Lambda_{3}\left(P_{2}, P_{3}, P_{4}\right)-[1,2]^{2}=0 .
$$

Since $\Lambda_{3}\left(P_{2}, P_{3}, P_{4}\right)>0$, it follows that $K\left(P_{1}, P_{2}, P_{3}, P_{4}\right) \leqq 0$. If every triple is linear, then the rank of $\Lambda_{4}\left(P_{1}, P_{2}, P_{3}, P_{4}\right)$ is two and, conse- 
quently, the rank of the determinant in (1) is three. Therefore, $K\left(P_{1}, P_{2}, P_{3}, P_{4}\right)=0$.

Corollary. If $P, Q, R, S$ is a quadruple of distinct points, then the three products, $\sinh (P Q / 2) \cdot \sinh (R S / 2), \sinh (P R / 2) \cdot \sinh (Q S / 2)$, $\sinh (P S / 2) \cdot \sinh (Q R / 2)$, of the hyperbolic sines of half the "opposite" distances satisfy the triangle inequality.

Proof. By Theorem 3.2, $K(P, Q, R, S) \leqq 0$. However, expansion of $K(P, Q, R, S)$ and routine but tedious computations yield $K$ $(P, Q, R, S)=A \cdot B \cdot C \cdot D$, where

$A=-[\sinh \lambda \sinh \delta+\sinh \varepsilon \sinh \theta+\sin \alpha \sinh \beta]$

$B=[\sinh \lambda \sinh \delta+\sinh \varepsilon \sinh \theta-\sin \alpha \sinh \beta]$

$C=[\sinh \lambda \sinh \delta-\sinh \varepsilon \sinh \theta+\sin \alpha \sinh \beta]$

$D=[-\sinh \lambda \sinh \delta+\sinh \varepsilon \sinh \theta+\sin \alpha \sinh \beta]$

and $\lambda=P Q / 2, \delta=R S / 2, \varepsilon=P R / 2, \theta=Q S / 2, \alpha=P S / 2$ and $\beta=Q R / 2$.

Since $K(P, Q, R, S) \leqq 0$ and no two of $B, C, D$ can be negative (consider their sum), it follows that $B, C, D$ are all nonnegative.

REMARK. The above corollary is the hyperbolic analogue of the ptolemaic inequality. It has been shown [3] that the ptolemaic inequality itself is valid in the hyperbolic plane.

Theorem 3.2. shows that $K(P, Q, R, S)$ vanishes if $P, Q, R, S$ are points on a line. Moreover, in view of Definition 3.1, we have shown for any four distinct points $P, Q, R, S$

$$
\{P S, Q R\}+\{P Q, R S\} \geqq 1 .
$$

Since $\sinh (x+y)=\sinh x \cosh y+\sinh y \cosh x$, it can be seen that if $P, Q, R, S$ lie on a line and occur in some cyclic permutation of the order $P Q R S$, then

$$
\{P S, Q R\}+\{P Q, R S\}=1 \text {. }
$$

Let $P R / / Q S$ denote the fact that $P, Q, R, S$ lie on a cycle and occur in some cyclic permutation of the order $P Q R S$.

THEOREM 3.3. If $P, Q, R, S$ are distinct points and if $P R / / Q S$ then $\{P S, Q R\}+\{P Q, R S\}=1$.

Proof. Theorem 3.2 and the above remarks show the validity of the theorem in case the cycle is a line. Suppose, then, that the four points lie on a horocycle, circle or equidistant curve. There exists an inversion which maps such a cycle onto a line. Thus, if $P^{\prime}, S^{\prime}, Q^{\prime}, R^{\prime}$ 
are the inverse points of $P, Q, R, S$ then $P^{\prime} R^{\prime} / / Q^{\prime} S^{\prime}$ and $\left\{P^{\prime} S^{\prime}, Q^{\prime} R^{\prime}\right\}$ $+\left\{P^{\prime} Q^{\prime}, R^{\prime} S^{\prime}\right\}=1$. Since cross ratio is an inversive invariant, $\{P S, Q R\}+\{P Q, R S\}=1$.

THEOREM 3.4. If $P, Q, R, S$ are four distinct points such that $\{P S, Q R\}+\{P Q, R S\}=1$ then $P R / / Q S$.

Proof. Three points of the hyperbolic plane lie on a line, circle, horocycle, or equidistant curve. We first suppose three of the points, say, $Q, R, S$ are collinear. Since $Q, R, S$ are collinear, $\Lambda_{3}(Q, R, S)=$ $0, K(Q, R, S) \neq 0$ and with the notation of Theorem 3.2, $I(Q, R, S)=0$. By hypothesis $K(P, Q, R, S)=0$, and $I(P, Q, R, S)=0$. It now follows that the rank of $I(P, Q, R, S)$ is three. Consequently, the rank of $\Lambda_{4}(P, Q, R, S)$ is two. Therefore, $\Lambda_{3}(P, Q, S)=0$ and $P, Q, S$ are collinear. It now follows that $P, Q, R, S$ are collinear and $P R / / Q S$.

If $Q, R, S$ lie on any cycle, this cycle may be mapped onto a line by an inversion. If $P^{\prime}, Q^{\prime}, R^{\prime}, S^{\prime}$ are inverse points of $P, Q, R, S$ then by the above, $P^{\prime} R^{\prime} / / Q^{\prime} S^{\prime}$. If the line of $P^{\prime}, Q^{\prime}, R^{\prime}, S^{\prime}$ is mapped back onto the cycle of $Q, R, S$ by the same inversion, it follows that $P R / / Q S$.

The following theorem, which is a hyperbolic analogue of Ptolemy's Theorem and its converse, has now been obtained.

Theorem 3.5. Four distinct points $P_{1}, P_{2}, P_{3}, P_{4}$ of the hyperbolic plane lie on a line, circle, horocycle, or one branch of an equidistant curve if and only if the determinant

$$
K\left(P_{1}, P_{2}, P_{3}, P_{4}\right)=\left|\sinh ^{2}\left(P_{i} P_{j} / 2\right)\right| \text { vanishes }(i, j=1,2,3,4) .
$$

In order to obtain the generalization of this analogue of Ptolemy's Theorem and its converse, we need the following lemma.

Lemma 3.6. If $P_{1}, P_{2}, P_{3}, P_{4}, P_{5}$ are five points then the determinant $K\left(P_{1}, P_{2}, P_{3}, P_{4}, P_{5}\right)=\left|\sinh ^{2}\left(P_{i} P_{j} / 2\right)\right|$ vanishes $(i, j=1,2,3,4,5)$.

Proof. Since the rank of $\Lambda_{5}\left(P_{1}, P_{2}, P_{3}, P_{4}, P_{5}\right)$ is less than or equal to three, it follows that the determinant, $H$, obtained from $\Lambda_{5}$ $\left(P_{1}, P_{2}, P_{3}, P_{4}, P_{5}\right)$ by bordering it with a first column and a first row with common element -1 , and the rest of the elements of the first column all ones and the rest of the element in the first row all zeroes has rank less than or equal to four. The determinant, $I$, obtained from $H$ by substracting the first column from the second, third, 
fourth, fifth and sixth columns, respectively, and then substituting $2 \sinh ^{2}(x / 2)$ for $\cosh (x)-1$ also has rank less than or equal to four. Hence $K\left(P_{1}, P_{2}, P_{3}, P_{4}, P_{5}\right)=0$.

Theorem 3.7. If $P_{1}, P_{2}, \cdots, P_{n}$ are $n$ pairwise distinct points of the hyperbolic plane, $(n \geqq 4)$, then necessary and sufficient condition that $P_{1}, P_{2}, \cdots, P_{n}$ lie on a line, circle, horocycle, or one branch of an equidistant curve is that the matrix

$$
K\left(P_{1}, P_{2}, \cdots, P_{n}\right)=\left|\sinh ^{2}\left(P_{i} P_{j} / 2\right)\right|(i, j=1,2, \cdots, n),
$$

have rank three.

Proof. Suppose $P_{1}, P_{2}, \cdots, P_{n}$ are pairwise distinct points of the hyperbolic plane which lie on a line, circle, horocycle, or one branch of an equidistant curve. Then the leading principal minor of order three of $K\left(P_{1}, P_{2}, \cdots, P_{n}\right)$ is nonzero, while the determinants obtained from this principal minor by adjoining one row and one column or two rows and two columns vanish by Theorem 3.3 and Lemma 3.6, respectively. It follows that the rank of $K\left(P_{1}, P_{2}\right.$, $\left.\cdots, P_{n}\right)$ is three.

Conversely, if the rank of $K\left(P_{1}, \cdots, P_{n}\right)$ is three then every principal minor of $K\left(P_{1}, P_{2}, \cdots, P_{n}\right)$ of order four is zero. It follows from Theorem 3.5 that each quadruple of the points and hence the $n$ points lie on a line, circle, horocycle, or one branch of an equidistant curve.

4. Circles, horocycles and equidistant curves. It is noted, in the hyperbolic analogue of Ptolemy's Theorem and its converse, that the relation satisfied by the mutual distances of four points is the same for four points on a line, circle, horocycle, and one branch of an equidistant curve. Since three points are collinear if and only if one of the distances determined by the three points is equal to the sum of the other two, the characterization of three collinear points is immediate. The purpose of this section is to find metric conditions which are necessary and sufficient to insure that three points lie on a circle, horocycle, or one branch of an equidistant curve, respectively. In this section the vertices of a triangle will be denoted $A, B, C$ and $a, b, c$ will denote the lengths of the sides opposite the vertices $A$, $B, C$ respectively.

Theorem 4.1. Suppose $A, B, C$ are vertices of a triangle. Then $A, B, C$ lie on a circle if and only if a real number $R$, the circumradius, exists such that $2 R \geqq \max \{a, b, c\}$ and the determinant 


$\alpha(A, B, C)=\left|\begin{array}{cccc}1 & \cosh c & \cosh b & 1 \\ \cosh c & 1 & \cosh a & 1 \\ \cosh b & \cosh a & 1 & 1 \\ 1 & 1 & 1 & \operatorname{sech}^{2} R\end{array}\right|=0$

Proof. Clearly, $A, B, C$ are points on a circle if and only if a point $T$ exists such that $T A=T B=T C=R$ and $2 R \geqq \max \{a, b, c\}$ by the triangle inequality. Since $\Lambda_{4}(A, B, C, T)=0$, it follows, by factoring $\cosh R$ from the last row and column of $\Lambda_{4}(A, B, C, T)$, that $\alpha(A, B, C)=0$.

Conversely, suppose $A, B, C$ are vertices of a triangle and a real number $R$ exists such that $\alpha(A, B, C)=0$ and $2 R \geqq \max \{a, b, c\}$. Then

(2) $\left|\begin{array}{cccc}1 & \cosh c & \cosh b & \cosh R \\ \cosh c & 1 & \cosh a & \cosh R \\ \cosh b & \cosh a & 1 & \cosh R \\ \cosh R & \cosh R & \cosh R & 1\end{array}\right|=0$

Two cases are to be considered.

Case 1. The distance $A B$ is less than $2 R$.

Since the function $A X$, ( $X$ on the perpendicular bisector $l$, of the segment joining $A$ and $B$ ), is continuous, monotone increasing, and is not bounded above as $X$ recedes along either half-line of $l$ determined by the midpoint of the segment joining $A$ and $B$, points $S, S^{\prime}$ of $l$ exist such that $A S=A S^{\prime}=R$. Also, $B S=A S=B S^{\prime}$. It follows that $C S \neq C S^{\prime}$, for otherwise $C$ would lie on the line joining $A$ and $B$, contrary to the fact that $A, B, C$ are noncollinear points.

Denote by $\Lambda(A, B, C, S ; x)$ the function obtained from $\Lambda_{4}(A, B$, $C, S$ ) by replacing $C S$ by $x$. This function is not identically zero, since the coefficient of $\cosh ^{2} x$ in the development of the determinant is $-\Lambda_{2}(A, B) \neq 0$. It follows that the function vanishes for at most two values of $x$. Since $A S=A S^{\prime}, B S=B S^{\prime}$ and $\Lambda_{4}(A, B, C, S)=\Lambda_{4}$ $\left(A, B, C, S^{\prime}\right)=0, C S$ are $C S^{\prime}$ are the distinct roots of $\Lambda(A, B, C, S ; x)$ $=0$. From (2), $R$ is also a root of $\Lambda(A, B, C, S ; x)=0$. Therefore, $C S=R$ or $C S^{\prime}=R$ and $A, B, C$ lie on a circle with circumradius $R$.

Case 2. The distance $A B$ is equal to $2 R$.

Let $S$ be the midpoint of the segment joining $A$ and $B$. Then $A S=B S=R$. Again, denote by $\Lambda(A, B, C, S ; x)$ the function obtained 
from $\Lambda_{4}(A, B, C, S)$ by replacing $C S$ by $x$. By an expansion theorem for determinants

$$
\Lambda(A, B, C, S ; x)=\left[\Lambda_{3}(A, B, C) \Lambda_{3}(A, B, S)-[\cosh x]^{2}\right] / \Lambda_{2}(A, B),
$$

where $[\cosh x]$ denotes the cofactor of the element $\cosh x$ in the determinant $\Lambda(A, B, C, S ; x)$. Hence, since $\Lambda_{3}(A, B, S)=0$, the equation $\Lambda(A, B, C, S ; x)=0$ has only one root. From (2), $R$ is a root of this equation, while $\Lambda_{4}(A, B, C, S)=0$ by the Preliminary Remarks. Therefore, $C S=R$ and $A, B, C$ lie on a circle with circumradius $R$.

Theorem 4.2. If $A, B, C$ are vertices of a triangle, then $A, B, C$ lie on a horocycle if and only if

$$
\beta(A, B, C)=\left|\begin{array}{cccc}
1 & \cosh c & \cosh b & 1 \\
\cosh c & 1 & \cosh a & 1 \\
\cosh b & \cosh a & 1 & 1 \\
1 & 1 & 1 & 0
\end{array}\right|=0
$$

Proof. Subtracting the first column of $(A, B, C)$ from the second, third, and fourth columns, respectively, and making use of the fact that $\cosh (x)-1=\sinh ^{2}(x / 2)$; we have:

$\beta(A, B, C)=4 \cdot \lambda \cdot \delta \cdot \varepsilon \cdot \theta$ where

$$
\begin{aligned}
& \lambda=\sinh a / 2-\sinh b / 2-\sinh c / 2 \\
& \delta=\sinh a / 2-\sinh b / 2+\sinh c / 2 \\
& \varepsilon=\sinh a / 2+\sinh b / 2-\sinh c / 2 \\
& \theta=\sinh a / 2+\sinh b / 2+\sinh c / 2 .
\end{aligned}
$$

Hence, $\beta(A, B, C)=0$ if and only if one of the numbers $\sinh \alpha / 2$, $\sinh b / 2, \sinh c / 2$ is the sum of the other two. It follows from the formula for the length of arc of a horocycle that $\beta(A, B, C)=0$ if $A, B, C$ are points on a horocycle.

Conversely, if $\beta(A, B, C)=0$, then no generality is lost if we assume $\varepsilon=0$. Then $\sinh a / 2+\sinh b / 2=\sinh c / 2$, and $c$ is the greatest of $a, b, c$. Since

$\sinh (a+b) / 2=\sinh a / 2 \cosh b / 2+\sinh b / 2 \cosh a / 2>\sinh c / 2$,

$a+b>c$. Thus, a triangle $A, B, C$ exists such that $C$ lies one of the two horocyclic arcs that join $A$ and $B$. Thus, if $\beta(A, B, C)=0$ then $A, B, C$ lie on a horocycle.

If $a=b=c$, then $\beta(A, B, C)=-3(\cosh c-1)^{2}$, and it follows by continuous variation that $\beta(A, B, C)<0$ whenever $A, B, C$ lie on a circle, and $\beta(A, B, C)>0$ whenever $A, B, C$ lie on one branch of an equidistant curve. 
As a result of Theorems 4.1 and 4.2, the following theorem is immediate.

Theorem 4.3. If $A, B, C$ are vertices of a triangle, then $A, B, C$ lie on one branch of an equidistant curve if and only if for each real number $R \geqq 1 / 2 \max \{a, b, c\}, \alpha(A, B, C) \neq 0 \neq \beta(A, B, C)$.

The author wishes to thank Professor L.M. Blumenthal for the suggestion of the problem and for his helpful criticism. The author also wishes to thank H.S.M. Coxeter for his suggestion to use Poincarés model. The proofs of some of the theorems given here are due to his helpful suggestions. Moreover, Theorem 3.1 is due to him.

\section{REFERENCES}

1. L. M. Blumenthal, Theory and applications of distance geometry, The Clarendon Press, Oxford, 1953.

2. H. S. M. Coxeter and S. L. Greitzer, Geometry revisited, Random House Inc., New York, 1967.

3. R. W. Freese, Ptolemaic Metric Spaces, doctoral dissertation, University of Missouri, Columbia, 1961.

4. J. Haantjes, A characteristic local property of Geodesics in certain metric spaces, Proc. Akad. Wetensch., Amsterdam, 50 (1947), 496-508.

5. — De Stelling von Ptolemeus, Simon Stevin 29 (1952), 25-31

6. T. Kubota, On the extended Ptolemy's theorem in hyperbolic geometry, Sci Rep. Tohoku Univ. (I) 2, 3 (1912), 131-156.

7. Z. Kurnik, and V. Volenec, Glasnik Matematicki, Tom 2 22, No. 2-1967.

8. D. M. Y. Sommerville, The elements of non-Euclidean geometry, G. Bell and Sons, LTD., London, 1914.

9. Harold E. Wolfe, Introduction to non-Euclidean geometry, Holt, Rinehart and Winston, New York, 1945.

Received August 4, 1969. The results in this paper are contained in the author's doctoral dissertation at the University of Missouri at Columbia.

Utah State University 



\section{PACIFIC JOURNAL OF MATHEMATICS}

\section{EDITORS}

H. SAMELSON

Stanford University

Stanford, California 94305

\section{Richard Pierce}

University of Washington

Seattle, Washington 98105
J. DugundJI

Department of Mathematics

University of Southern California

Los Angeles, California 90007

RICHARD ARENS

University of California

Los Angeles, California 90024

\section{ASSOCIATE EDITORS}

\section{E. F. BeCKenBACH}

B. H. NeUmanN
K. YosHida

\section{SUPPORTING INSTITUTIONS}

\author{
UNIVERSITY OF BRITISH COLUMBIA \\ CALIFORNIA INSTITUTE OF TECHNOLOGY \\ UNIVERSITY OF CALIFORNIA \\ MONTANA STATE UNIVERSITY \\ UNIVERSITY OF NEVADA \\ NEW MEXICO STATE UNIVERSITY \\ OREGON STATE UNIVERSITY \\ UNIVERSITY OF OREGON \\ OSAKA UNIVERSITY \\ UNIVERSITY OF SOUTHERN CALIFORNIA
}

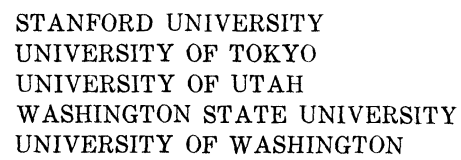

STANFORD UNIVERSITY UNIVERSITY OF TOKYO

UNIVERSITY OF UTAH

WASHINGTON STATE UNIVERSITY

UNIVERSITY OF WASHINGTON

The Supporting Institutions listed above contribute to the cost of publication of this Journal, but they are not owners or publishers and have no responsibility for its content or policies.

Mathematical papers intended for publication in the Pacific Journal of Mathematics should be in typed form or offset-reproduced, (not dittoed), double spaced with large margins. Underline Greek letters in red, German in green, and script in blue. The first paragraph or two must be capable of being used separately as a synopsis of the entire paper. The editorial "we" must not be used in the synopsis, and items of the bibliography should not be cited there unless absolutely necessary, in which case they must be identified by author and Journal, rather than by item number. Manuscripts, in duplicate if possible, may be sent to any one of the four editors. Please classify according to the scheme of Math. Rev. Index to Vol. 39. All other communications to the editors should be addressed to the managing editor, Richard Arens, University of California, Los Angeles, California, 90024.

50 reprints are provided free for each article; additional copies may be obtained at cost in multiples of 50 .

The Pacific Journal of Mathematics is published monthly. Effective with Volume 16 the price per volume (3 numbers) is $\$ 8.00$; single issues, $\$ 3.00$. Special price for current issues to individual faculty members of supporting institutions and to individual members of the American Mathematical Society: $\$ 4.00$ per volume; single issues $\$ 1.50$. Back numbers are available.

Subscriptions, orders for back numbers, and changes of address should be sent to Pacific Journal of Mathematics, 103 Highland Boulevard, Berkeley, California, 94708.

PUBLISHED BY PACIFIC JOURNAL OF MATHEMATICS, A NON-PROFIT CORPORATION

Printed at Kokusai Bunken Insatsusha (International Academic Printing Co., Ltd.), 7-17, Fujimi 2-chome, Chiyoda-ku, Tokyo, Japan. 


\section{Pacific Journal of Mathematics}

\section{Vol. 34, No. 3 \\ July, 1970}

Richard Hindman Bouldin, The peturbation of the singular spectrum

Hugh D. Brunk and Søren Glud Johansen, A generalized Radon-Nikodym derivative .

Henry Werner Davis, F. J. Murray and J. K. Weber, Families of $L_{p}$-spaces

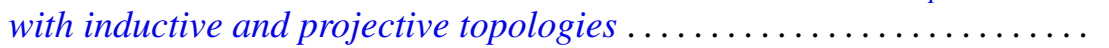

Esmond Ernest Devun, Special semigroups on the two-cell .

Murray Eisenberg and James Howard Hedlund, Expansive automorphisms

of Banach spaces ......................................

Frances F. Gulick, Actions of functions in Banach algebras.

Douglas Harris, Regular-closed spaces and proximities.

Norman Lloyd Johnson, Derivable semi-translation planes . .

Donald E. Knuth, Permutations, matrices, and generalized Young

tableaux..........................................

Herbert Frederick Kreimer, Jr., On the Galois theory of separable

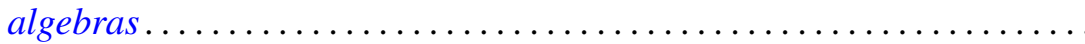

You-Feng Lin and David Alon Rose, Ascoli's theorem for spaces of

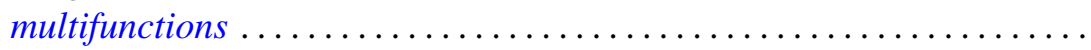

David London, Rearrangement inequalities involving convex functions . . . .

Louis Pigno, A multiplier theorem.

749

Helga Schirmer, Coincidences and fixed points of multifunctions into trees.

755

Richard A. Scoville, Some measure algebras on the integers .

Ralph Edwin Showalter, Local regularity of solutions of Sobolev-Galpern

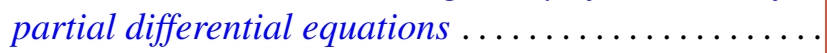

Allan John Sieradski, Twisted self-homotopy equivalences

John H. Smith, On S-units almost generated by S-units of subfields ...

803

Masamichi Takesaki, Algebraic equivalence of locally normal

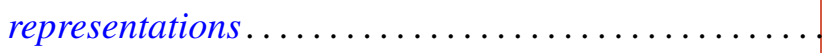

Joseph Earl Valentine, An analogue of Ptolemy's theorem and its converse in

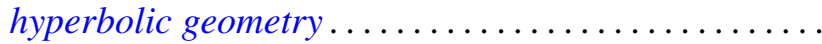

David Lawrence Winter, Solvability of certain p-solvable linear groups of finite order 